\title{
Dose Preparatória de Atracúrio: Aferição da Fadiga no Músculo Orbicular do Olho e Condições de Intubação Orotraqueal *
}

\section{Priming Dose of Atracurium: Measuring Orbicularis Oculi Muscle Fade and Tracheal Intubation Conditions}

\author{
Giovani de Figueiredo Locks ${ }^{1}$; Maria Cristina Simões de Almeida, TSA ${ }^{2}$
}

\begin{abstract}
RESUMO
Locks GF, Almeida MCS - Dose Preparatória de Atracúrio: Aferição da Fadiga no Músculo Orbicular do Olho e Condições de Intubação Orotraqueal
\end{abstract}

JUSTIFICATIVA E OBJETIVOS: O método da dose preparatória (priming principle) consiste na injeção de uma pequena dose do bloqueador neuromuscular, minutos antes da administração do restante da dose preconizada para intubação traqueal, para encurtar o início de ação dos bloqueadores neuromusculares (BNM) adespolarizantes. Existe, porém, possibilidade de fadiga muscular e broncoaspiração. De interesse especial para as manobras de intubação orotraqueal e proteção das vias aéreas, são os músculos da laringe. Como sua monitorização direta impõe dificuldades técnicas, fo demonstrado que o músculo orbicular do olho apresenta correlação com os músculos laríngeos quanto à sensibilidade aos BNM. O objetivo desta pesquisa é avaliar se há fadiga do músculo orbicular ocular após o uso da dose preparatória de atracúrio e comparar as condições clínicas de intubação traqueal após dois intervalos entre as doses.

MÉTODO: Foram selecionados 35 pacientes adultos, estado físico ASA I ou II, sem fatores de risco para broncoaspiração, submetidos à cirurgia eletiva. Foi induzida anestesia geral com alfentanil e propofol e os pacientes foram ventilados manualmente sob máscara. Em seguida, foram posicionados os eletrodos para estimular o ramo temporal do nervo facial e o transdutor de aceleração no músculo orbicular do olho. Administrou-se a dose preparatória de atracúrio $\left(0,02 \mathrm{mg} \mathrm{kg}^{-1}\right)$ e a relação $T_{4} / T_{1}$ foi avaliada a cada minuto durante 5 minutos em 20 casos (G1) e 7 minutos em 13 casos (G2). Após este intervalo, administrou-se a dose complementar de atracúrio $\left(0,5 \mathrm{mg} \cdot \mathrm{kg}^{-1}\right)$ e procedeu-se a intubação orotraqueal após um minuto. Foi considerada fadiga clinicamente importante a relação $T_{4} / T_{1}$ inferior a 0,9 .

\footnotetext{
* Recebido do (Received from) Hospital Universitário Polydoro Ernani de San Thiago, Universidade Federal de Santa Catarina (UFSC), Florianópolis, SC

1. $M E_{2}$ do Centro Integrado de Treinamento da Secretaria Estadual de Saúde, Florianópolis, SC

2. Doutora pela Universidade Johanes Gutenberg e Professora Adjunta da UFSC
}

Apresentado (Submitted) em 18 de dezembro de 2002 Aceito (Accepted) para publicação em 25 de março de 2003

Endereço para correspondência (Correspondence to) Dr. Giovani de Figueiredo Locks

Rua Heidelberg, 125 Córrego Grande

88037-390 Florianópolis, SC

(c) Sociedade Brasileira de Anestesiologia, 2003
RESULTADOS: Nenhum paciente apresentou fadiga durante 0 intervalo de monitorização. Em $80 \%$ e $69 \%$ dos pacientes de G1 ou G2, respectivamente, as condições de intubação traqueal foram consideradas clinicamente aceitáveis ( $p>$ $0,05)$

CONCLUSÕES: A dose preparatória de atracúrio (0,02 $\mathrm{mg} \cdot \mathrm{kg}^{-1}$ ) não causa fadiga no músculo orbicular do olho e não há diferença entre aguardar 5 ou 7 minutos como intervalo entre as doses do bloqueador neuromuscular.

Unitermos: BLOQUEADORES NEUROMUSCULARES, Não-despolarizante: atracúrio; INTUBAÇÃO TRAQUEAL; MONITORIZAÇÃO: aceleromiografia

\section{SUMMARY}

Locks GF, Almeida MCS - Priming Dose of Atracurium: Measuring Orbicularis Oculi Muscle Fade and Tracheal Intubation Conditions

BACKGROUND AND OBJECTIVES: The priming principle consists of administering a low neuromuscular blocker dose, minutes before the total dose for tracheal intubation, to shorten non-depolarizing blockers onset. There is, however, the risk for muscle fade and bronchoaspiration. Laryngeal muscles are of especial interest for tracheal intubation maneuvers and airway protection. Since their direct monitoring imposes technical difficulties, it has been reported that orbicularis oculi correlates with laryngeal muscles in terms of sensitivity to neuromuscular blocks. This study aimed at evaluating the presence of orbicularis oculi muscle fade after priming atracurium dose and at comparing clinical tracheal intubation conditions after two priming dose intervals.

METHODS: Participated in this study 35 adult patients, physical status ASA I or II, without risk factors for bronchoaspiration and submitted to elective surgeries. General anesthesia was induced with alfentanil and propofol and patients were manually ventilated under mask. Surface electrodes were then positioned on the temporal branch of the facial nerve, and the acceleration transducer was placed on the orbicularis oculi. Priming atracurium dose $\left(0.02 \mathrm{mg} . \mathrm{kg}^{-1}\right)$ was administered and $T_{4} / T_{1} \mathrm{ra}-$ tio was evaluated every minute during 5 minutes in 20 cases (G1) and during 7 minutes in 13 cases (G2). After this interval, complementary atracurium dose $\left(0.5 \mathrm{mg}^{\mathrm{kg}} \mathrm{g}^{-1}\right)$ was administered and tracheal intubation was performed one minute later. Fade was defined as $T_{4} / T_{1}$ ratio below 0.9 .

RESULTS: There has been no fade in any patient during the monitoring interval. In $80 \%$ and $69 \%$ of $G 1$ or $G 2$ patients, respectively, tracheal intubation was classified as clinically acceptable $(p>0.05)$.

CONCLUSIONS: Priming atracurium dose $\left(0.02 \mathrm{mg}^{\mathrm{kg}} \mathrm{kg}^{-1}\right)$ does not determine orbicularis oculi fade and there is no difference between 5 or 7 minutes priming intervals.

Key Words: MONITORING: acceleromyography; NEUROMUSCULAR BLOCKERS, Non-depolarizing: atracurium; TRACHEAL INTUBATION 


\section{INTRODUÇÃO}

$\mathrm{N}_{\mathrm{s} a}^{\mathrm{as}}$ as situações de estômago cheio, o intervalo entre a perda da consciência e a intubação traqueal deve ser o mais curto possível. Nesses casos são usados bloqueadores neuromusculares (BNM) de rápido início de ação. A succinilcolina é o BNM disponível comercialmente que apresenta menor tempo de início de ação, mas efeitos colaterais limitam sua indicação ${ }^{1}$. Os substitutos, os BNM adespolarizantes, apresentam como inconveniente um tempo de instalação longo no qual o paciente fica exposto ao risco de broncoaspiração e hipóxia ${ }^{2}$. Para minimizar o problema da latência longa foi idealizado o método da dose preparatória (priming principle $)^{3}$. Esse método pressupõe a injeção de uma fração da dose do BNM adespolarizante programado para uso na anestesia, minutos antes da administração do restante da dose preconizada para intubação traqueal. Miller ${ }^{4}$, em editorial já na década de 1980, levantava muitas questões sobre os benefícios e os riscos da dose preparatória. Dentre elas, pode-se destacar a magnitude da dose. Se a dose preparatória for insuficiente, ela não será eficaz em promover condições adequadas de intubação orotraqueal e, se for excessiva, implicará em fadiga muscular. Outros questionamentos dizem respeito ao intervalo ideal entre a dose preparatória e a dose para intubação. Até o presente, essas questões permanecem sem resposta definitiva.

É de conhecimento estabelecido que cada grupo muscular reage diferentemente aos $\mathrm{BNM}^{5}$. De interesse especial para as manobras de intubação traqueal é o perfil de relaxamento dos músculos da laringe. A monitorização da função neuromuscular diretamente nesses músculos, no entanto, necessita de equipamentos especiais e sofisticados sendo limitada à pesquisa. Após estudos com diversos músculos, observou-se que o músculo orbicular do olho apresenta um comportamento similar ao dos músculos da laringe. Asua monitorização oferece, então, bons subsídios para indicar o melhor momento de se intubar a traquéia, mais do que quando se emprega métodos tradicionais de aferição do relaxamento no músculo adutor do polegar ${ }^{6}$.

Este estudo tem como objetivo abordar dois pontos controversos no princípio de dose preparatória: a presença de fadiga no músculo orbicular do olho como um reflexo do comportamento dos músculos da laringe, e o intervalo entre a primeira dose e a dose complementar do relaxante. Como uma conseqüência da finalidade do método, é também objetivo avaliar as condições de intubação após dois intervalos de tempo pré-determinados.

\section{MÉTODO}

Após aprovação do protocolo pela Comissão de Ética da Instituição e obtenção do consentimento informado, foram selecionados 35 pacientes de ambos os sexos com idades entre 18 e 65 anos, estado físico ASA I ou II, submetidos à cirurgia eletiva.

Foram excluídos da pesquisa os pacientes que se recusaram a participar do estudo, os portadores de doença renal, hepáti- ca ou neuromuscular, os que tinham fatores de previsão para intubação difícil e os que estavam em uso de medicação que sabidamente altera a função neuromuscular. Foram também excluídos gestantes ou mulheres em período de aleitamento, obesos, portadores de Diabetes Mellitus, de hérnia de hiato, de grandes massas abdominais, situações clínicas que podem implicar em broncoaspiração.

Os pacientes receberam como medicação pré-anestésica, midazolam $(7,5 \mathrm{mg})$ por vía oral 90 minutos antes da anestesia. Na sala de operações, após acesso venoso convencional, os pacientes foram monitorizados com cardioscópio em $D_{\|}$, oxímetro de pulso e medida da pressão arterial não invasiva a cada três minutos. Após administração de oxigênio a $100 \%$ sob máscara durante 3 minutos, a indução da anestesia foi feita com alfentanil $\left(50 \mu \mathrm{g} \cdot \mathrm{kg}^{-1}\right)$ e propofol $(2,5$ $\left.\mathrm{mg} \cdot \mathrm{kg}^{-1}\right)$. Em seguida, foram posicionados os eletrodos da monitorização neuromuscular na região pré-auricular e o transdutor de aceleração no músculo orbicular do olho. Doses complementares de $50 \mathrm{mg}$ de propofol foram administradas conforme necessidade e o paciente, ventilado manualmente sob máscara. Administrou-se a dose preparatória de atracúrio $\left(0,02 \mathrm{mg} \cdot \mathrm{kg}^{-1}\right)$, e a transmissão neuromuscular foi avaliada a cada minuto, sendo registrado o valor da relação $\mathrm{T}_{4} / \mathrm{T}_{1}$. Os pacientes foram divididos aleatoriamente em dois grupos. Em 20 pacientes (grupo 1 - G1), o intervalo entre a dose preparatória e a dose complementar de intubação traqueal foi de cinco minutos. Em 13 (grupo 2-G2), esse intervalo foi de sete minutos. Após o intervalo estabelecido foi administrada a dose complementar de intubação de atracúrio $(0,5$ $\left.\mathrm{mg} \cdot \mathrm{kg}^{-1}\right)$, seguida de intubação orotraqueal após um minuto. As condições de intubação foram avaliadas segundo escala clínica $^{7}$.

As condições de laringoscopia foram consideradas:

Fácil: se os músculos masseteres estivessem relaxados e sem resistência à laringoscopia;

Regular: se os músculos masseteres estivessem parcialmente relaxados e com resistência à laringoscopia;

Difícil: se os músculos masseteres não estivessem relaxados e o paciente resistente ativamente à laringoscopia.

As condições de intubação traqueal foram consideradas:

Excelentes: se todos os itens avaliados fossem classificados como excelentes;

Boas: se todos os itens fossem classificados como excelentes ou bons;

Ruins: se um único ou mais itens fossem classificados como ruim.

Foi considerada fadiga muscular a relação $T_{4} / T_{1}$ inferior a 0,9 durante o intervalo de monitorização.

Os dados são apresentados como média e desvio padrão. Para análise estatística foram utilizados teste $t$ de Student para comparar os dados demográficos, e teste Exato de Fisher para comparação das condições de intubação traqueal. Valor de $p<0,05$ foi considerado como significativo. 


\section{RESULTADOS}

Foram incluídos 22 e 13 pacientes em G1e G2, respectivamente. Em dois casos do G1, houve perda do sinal da monitorização, visto que o aparelho utilizado calcula a relação $T_{4} / T_{1}$ apenas quando o sinal $\mathrm{T}_{1}$ é considerado superior a $20 \%$. Nesses dois casos, a resposta basal era superior a $20 \%$ e após a dose preparatória, não se obteve mais um sinal confiável. Esses casos foram excluídos do estudo estatístico.

A tabela I ilustra os dados demográficos dos pacientes em cada grupo. Não houve diferença estatística no que se refere a sexo, idade e do índice de massa corporal entre os grupos.

Tabela I - Dados Demográficos

\begin{tabular}{lcc}
\hline Grupo & $\mathrm{G} 1$ & $\mathrm{G} 2$ \\
\hline Masculino/Feminino & $7 / 13$ & $7 / 6$ \\
Idade (anos) * & $42,8 \pm 12,3$ & $39,2 \pm 10,8$ \\
IMC (kg.m ${ }^{-2}$ * & $24,6 \pm 4,6$ & $25,2 \pm 3,3$ \\
\hline
\end{tabular}

*Valores expressos pela Média \pm DP

Os registros obtidos da relação $T_{4} / T_{1}$, desde a resposta inicial e até os 5 ou 7 minutos de monitorização, estão apresentados na figura 1. Em momento nenhum do intervalo de monitorização, considerando as variáveis analisadas, houve detecção de fadiga.

As condições de intubação foram classificadas e comparadas conforme escala clínica no $\mathrm{G} 1$ e no $\mathrm{G} 2$, sendo que não houve diferença estatisticamente significativa entre os grupos ( $p=$ $0,64)$ (Tabela II e Figura 2). A grande maioria dos pacientes $(73,3 \%)$ apresentou condições clinicamente aceitáveis de intubação orotraqueal. Das variáveis que implicaram um conceito clinicamente inaceitável de intubação orotraqueal, assumem maior importância clínica para as manobras de intubação traqueal, as que são relacionadas à laringoscopia difícil e às cordas vocais fechadas. Essas condições foram anotadas em 2 pacientes em G1 e em 1 paciente em G2.

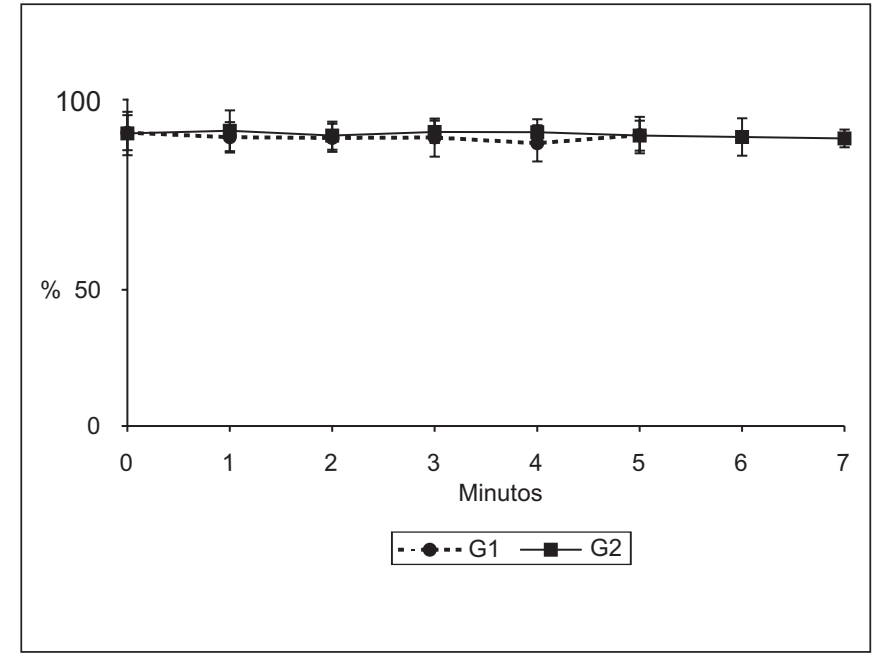

Figura 1 - Médias da Relação $T_{4} / T_{1}$ no Intervalo de Monitorização em G1 e G2

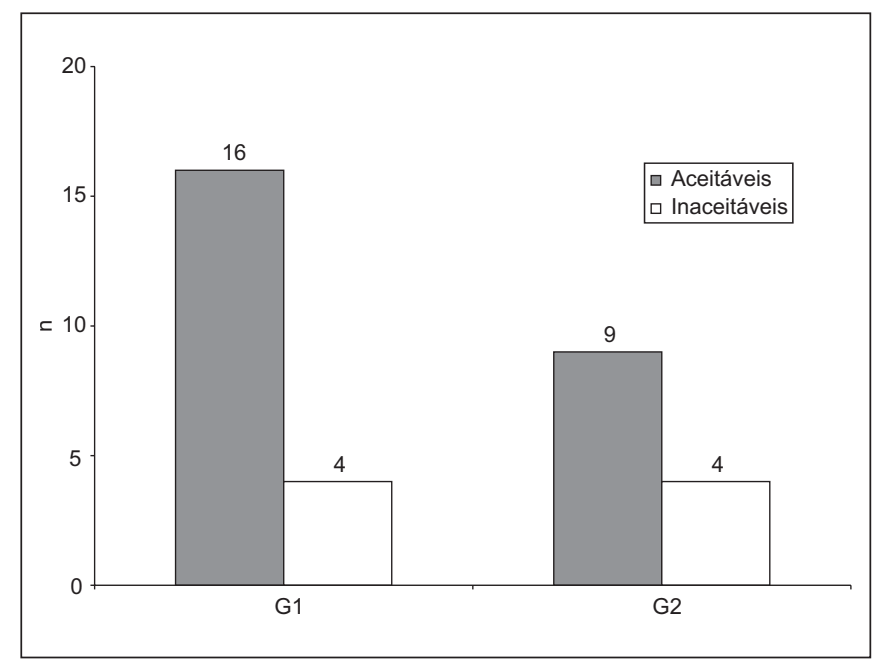

Figura 2 - Condições Clínicas de Intubação Traqueal em G1 e G2

Tabela II - Número de Pacientes em G1 e G2 (G1/G2) Classificados de Acordo com as Condições Clínicas no Momento da Intubação Traqueal. Adaptado de Viby-Mogensen e col.

\begin{tabular}{|c|c|c|c|}
\hline \multirow[t]{2}{*}{ Variáveis } & \multicolumn{2}{|c|}{ Clinicamente Aceitáveis } & \multirow{2}{*}{$\frac{\text { Clinicamente Inaceitáveis }}{\text { Ruins }}$} \\
\hline & Excelentes & Boas & \\
\hline Laringoscopia & $\begin{array}{l}\text { Fácil } \\
16 / 12\end{array}$ & $\begin{array}{l}\text { Regular } \\
2 / 2\end{array}$ & $\begin{array}{l}\text { Difícil } \\
1 / 0\end{array}$ \\
\hline \multicolumn{4}{|l|}{ Cordas Vocais } \\
\hline Posição & $\begin{array}{l}\text { Abduzidas } \\
12 / 10\end{array}$ & $\begin{array}{c}\text { Intermediária } \\
5 / 2\end{array}$ & $\begin{array}{l}\text { Fechadas } \\
\quad 0 / 1\end{array}$ \\
\hline Movimento & $\begin{array}{l}\text { Nenhum } \\
16 / 13\end{array}$ & $\begin{array}{l}\text { Presente } \\
\quad 1 / 1\end{array}$ & $\begin{array}{l}\text { Cordas vocais fechadas } \\
\qquad 2 / 1\end{array}$ \\
\hline \multicolumn{4}{|l|}{ Reação ao tubo/balonete } \\
\hline Com movimento dos membros & $\underset{11 / 8}{\text { Nenhum }}$ & $\begin{array}{l}\text { Leve } \\
5 / 5\end{array}$ & $\begin{array}{l}\text { Vigoroso } \\
2 / 1\end{array}$ \\
\hline Com tosse & $\begin{array}{l}\text { Nenhuma } \\
4 / 4\end{array}$ & $\begin{array}{c}\text { Com movimento do diafragma } \\
12 / 8\end{array}$ & $\begin{array}{c}\text { Sustentada }>10 \mathrm{seg} \\
2 / 2\end{array}$ \\
\hline
\end{tabular}




\section{DISCUSSÃO}

O dado que mais chamou a atenção neste trabalho foi a ausência de fadiga muscular após o uso da dose preparatória, quando aferida no músculo orbicular do olho.

Afadiga muscular pode ser avaliada clinicamente ou através da monitorização da função neuromuscular. Manifesta-se clinicamente, entre outros sintomas, por visão borrada e dificuldade para engolir ${ }^{8}$ e há relatos de broncoaspiração após uso de dose preparatória ${ }^{9,10}$. Com métodos instrumentais, considera-se fadiga quando a relação $T_{4} / T_{1}$ é inferior a 1 . Quando utilizado acelerometria, Kopman e col. ${ }^{11}$ afirmam que uma relação $T_{4} / T_{1}$ inferior a 0,9 deva ser interpretada como recuperação neuromuscular incompleta em potencial, e não 0,7 como anteriormente preconizado .

O método da dose preparatória baseia-se em princípio farmacológico em dois locais distintos: o primeiro, envolvendo uma ação pré-sináptica e o segundo, um efeito pós-juncional ${ }^{12}$. O ocupação pré-sináptica de receptores nicotínicos neuronais por bloqueadores neuromusculares implica uma diminuição da liberação de acetilcolina e, como conseqüência, a segunda dose de bloqueador neuromuscular produz paralisia mais rapidamente. No entanto, autores ${ }^{13}$ mostraram através de monitorização da transmissão neuromuscular que a presença de fadiga não é essencial para a eficácia do método. Posteriormente, em trabalho experimental ${ }^{14}$, estudou-se o comportamento de uma preparação nervo-músculo na presença de dose preparatória, concluindo-se claramente que a eficácia do princípio de dose preparatória está relacionada à concentração da droga na junção neuromuscular no final do intervalo "priming" e não propriamente ao efeito pré-sináptico.

O objetivo deste trabalho em aferir fadiga não é o de justificar o mecanismo do princípio, mas de identificar um efeito indesejável do método, efeito esse que adiciona riscos de complicações sérias como a broncoaspiração.

Aação pós-juncional indica que as moléculas de BNM primeiramente injetadas ocupam receptores nicotínicos nos músculos de forma que, com a segunda dose, há um efeito imediato de relaxamento, pois é ultrapassada a "margem de segurança" ${ }^{15}$. A "margem de segurança da transmissão neuromuscular" é um conceito que afirma que, para haver prejuízo na função neuromuscular, é necessária a ocupação de pelo menos $75 \%$ dos receptores da junção neuromuscular. Esta quantidade de receptores ocupados, a princípio, não deve ser alcançada pela dose preparatória, visto que o objetivo não é o relaxamento muscular.

Adose necessária para a ocupação de $75 \%$ dos receptores é muito variável. A monitorização da função neuromuscular, no entanto, não pode avaliar a ocupação de receptores na placa motora, de forma que nem com estimuladores de nervos e nem clinicamente se pode constatar quando a margem de segurança é ultrapassada, a não ser na sua forma extrema com o relaxamento muscular.

Existe muita controvérsia sobre a magnitude da dose preparatória a ser utilizada. Alguns autores ${ }^{2,8,16,17}$ comentam que doses iguais ou acima de $10 \%$ da dose efetiva $95\left(D E_{95}\right)$ acar- retam risco de causar fadiga. Recentemente Kopman e col. ${ }^{18}$ publicaram, em estudo com simulação computadorizada, que raramente a dose $10 \%$ da $\mathrm{DE}{ }_{95}$ causa fadiga, mas que 1 em cada 50 pacientes apresenta fadiga com a dose de $10 \%$ da dose de intubação traqueal. O presente estudo corrobora as afirmações no que diz respeito à segurança da dose preparatória de $10 \%$ da $\mathrm{DE}_{95}$, visto que não foi constatada fadiga em nenhum paciente.

Outro dado que chamou a atenção foi que não houve diferença estatisticamente significativa entre os grupos no que tange às condições de intubação traqueal ao variar o intervalo entre as doses do método empregado.

De acordo com a literatura, o tempo de instalação do bloqueio neuromuscular após agentes não despolarizantes é inversamente proporcional à dose empregada ${ }^{19}$, mas para doses sub-paralizantes, o tempo para alcançar o efeito máximo é relativamente constante. Hoje, sabe-se que esse tempo é de cerca de 6 minutos para cisatracúrio, 3 minutos para rocurônio e 5 minutos para vecurônio ${ }^{18}$. Para o atracúrio, estima-se que seja de 5,91 $\pm 0,34$ minutos $^{13}$, tempo no qual o efeito da dose preparatória tem benefício máximo em reduzir o tempo de instalação do bloqueio neuromuscular e promover melhores condições de intubação. Como a taxa de sucesso de intubação traqueal em G1 e G2 foi estatisticamente semelhante, este estudo sugere que 5 minutos é um intervalo adequado para ser usado entre as doses de atracúrio.

De forma geral, as condições de intubação nesse estudo foram aceitáveis após um minuto da administração da dose complementar de intubação de atracúrio. Valores semelhantes foram descritos por Schwarz e col. ${ }^{3}$, utilizando diversas modalidades de dose preparatória.

Em 8 casos a intubação orotraqueal foi classificada como clinicamente inaceitável, embora se tenha conseguido intubar todos os pacientes Atribuímos a alta taxa de pacientes que reagiram ao tubo/balonete ao tempo relativamente longo de monitorização sob anestesia superficial. Naguib e col. ${ }^{20}$ referem melhora nas condições de intubação traqueal após dose preparatória em estudo semelhante a este, utilizando incrementos de tiopental antes da intubação, confirmando o conceito de que o relaxante muscular não é o único fator necessário para o sucesso das manobras de laringoscopia e intubação traqueal ${ }^{21}$. A ausência de fadiga com a dose empregada, e condições nem sempre satisfatórias de intubação traqueal apontam justamente os dois pontos discutíveis do método da dose preparatória: a dose que protege a via aérea da broncoaspiração pode não ser suficiente para obtenção de sucesso na intubação traqueal. Apesar da intubação ter sido realizada em todos os casos, a presença de itens como cordas vocais fechadas e laringoscopia difícil deve ser apreciada e considerada como ponto falho e limitação do método. Nos pacientes com risco de broncoaspiração, deve-se avaliar criteriosamente os riscos que esta técnica pode proporcionar ao paciente.

Como conclusão, pode-se apontar que a dose preparatória de $0,02 \mathrm{mg} \cdot \mathrm{kg}^{-1}$ de atracúrio $\left(10 \%\right.$ da $\left.\mathrm{DE}_{95}\right)$ não causa fadiga no músculo orbicular do olho em até sete minutos, e não há 
diferença estatística entre as condições de intubação traqueal ao se aguardar 5 ou 7 minutos como intervalo entre a dose preparatória e a dose complementar de intubação.

\section{Priming Dose of Atracurium: Measuring Orbicularis Oculi Muscle Fade and Tracheal Intubation Conditions}

Giovani de Figueiredo Locks, M.D.; Maria Cristina Simões de Almeida, TSA, M.D.

\section{INTRODUCTION}

In situations where there is gastric content, the interval between consciousness loss and tracheal intubation should be as short as possible. Fast onset neuromuscular blockers (NMB) are used in such cases. Succinylcholine is the commercially available NMB with the shortest onset, however its indication is limited by side-effects ${ }^{1}$. Substitutes, or non-depolarizing NMBs, have the inconvenient of longer onset during which patients are exposed to the risk of bronchoaspiration and hypoxia ${ }^{2}$. The priming principle was developed to minimize long onset ${ }^{3}$. This method consists of the injection of a fraction of the non-depolarizing NMB scheduled for anesthesia, minutes before the administration of the remaining dose preconized for tracheal intubation. Miller ${ }^{4}$, in an editorial in the $80 \mathrm{~s}$, has raised several questions about priming dose benefits and risks, among them the magnitude of the dose. If the priming dose is insufficient, it will not be effective in promoting adequate tracheal intubation conditions and, if excessive, will imply muscle fade. Other questions are related to the optimal interval between priming and intubation doses. To date, these questions have not been definitely answered.

It is well known that each muscle group reacts differently to $\mathrm{NMBs}^{5}$. Laryngeal muscles relaxation profile is of special interest for tracheal intubation maneuvers. Direct neuromuscular function monitoring in those muscles, however, requires special and sophisticated equipment and is limited to research. After studying several muscles, it has been observed that the orbicularis oculi behaves similarly to laryngeal muscles. Its monitoring, then, offers good subsidies to indicate the best moment for tracheal intubation, being better than traditional methods checking adductor pollicis relaxation ${ }^{6}$.

This study aimed at addressing two controversial priming principle points: the presence of orbicularis oculi fade as a reflex of laryngeal muscles behavior, and the interval between priming and complementary blocker doses. As a consequence of the method's objective, our aim was also to evaluate intubation conditions after two predetermined intervals.

\section{METHODS}

After the Institution's Ethics Committee approval and their informed consent, participated in this study 35 patients of both genders, aged 18 to 65 years, physical status ASA I or II, submitted to elective surgeries.

Exclusion criteria were patients refusing to participate, patients with kidney, liver or neuromuscular disease, those with factors anticipating difficult intubation and patients under drugs knowingly affecting neuromuscular function. Pregnant and breast-feeding women, obese, diabetes mellitus or hiatal hernia patients, those with large abdominal mass and clinical situations which could imply bronchoaspiration were also excluded. Patients were premedicated with oral midazolam $(7.5 \mathrm{mg}) 90$ minutes before anesthesia. In the operating room and after conventional venous access, patients were monitored with cardioscopy in $D_{\|}$lead, pulse oximetry and non-invasive blood pressure at 3-minute intervals. After $100 \%$ oxygen administration under mask for 3 minutes, anesthesia was induced with alfentanil $\left(50 \mu \mathrm{g} \cdot \mathrm{kg}^{-1}\right)$ and propofol $\left(2.5 \mathrm{mg} \cdot \mathrm{kg}^{-1}\right)$. Then, neuromuscular monitoring electrodes were placed on the pre-auricular region and acceleration transducer on the orbicularis oculi. Additional $50 \mathrm{mg}$ propofol doses were administered as needed and patients were manually ventilated under mask. Priming atracurium dose $\left(0.02 \mathrm{mg} \cdot \mathrm{kg}^{-1}\right)$ was administered and neuromuscular transmission was evaluated at 1-minute intervals being recorded $T_{4} / T_{1}$ ratio. Patients were randomly distributed in two groups. In 20 patients (Group 1-G1), the interval between priming and complementary tracheal intubation dose was 5 minutes. In 13 patients (Group 2 - G2) this interval was 7 minutes. After the established interval, complementary atracurium intubation dose was administered $\left(0.5 \mathrm{mg} \cdot \mathrm{kg}^{-1}\right)$, followed by tracheal intubation 1 minute after. Intubation conditions were evaluated according to clinical scale ${ }^{7}$.

Laryngoscopy conditions were considered:

Easy: if masseter muscles were relaxed and without resistance to laryngoscopy;

Regular: if masseter muscles were partially relaxed and with resistance to laryngoscopy;

Difficult: if masseter muscles were not relaxed and patient actively resisted to laryngoscopy.

Intubation conditions were considered:

Excellent: if all evaluated items were classified as excellent;

Good: if all items were classified as excellent or good; Poor: if one or more items were classified as poor.

$\mathrm{T}_{4} / \mathrm{T}_{1}$ ratio below 0.9 during the monitoring interval was considered as muscle fade.

Data are shown in mean and standard deviation. Student's $t$ test was used to compare demographics and Fisher's Exact test was used to compare tracheal intubation conditions, considering significant $p<0.05$.

Revista Brasileira de Anestesiologia Vol. 53, N 6, Novembro - Dezembro, 2003 


\section{RESULTS}

There were 22 and 13 patients included in $\mathrm{G} 1$ and $\mathrm{G} 2$, respectively. Two $\mathrm{G} 1$ patients had monitoring signal loss, since the equipment used calculates $T_{4} / T_{1}$ ratio only when $T_{1}$ signal is considered above $20 \%$. In these 2 cases, baseline response was above $20 \%$ and after the priming dose no reliable signal was obtained. These cases were excluded from statistical analysis.

Table I shows demographics data for each group. There were no statistically significant differences in gender, age and body mass index between groups.

\section{Table I - Demographics Data}

\begin{tabular}{lcc}
\hline Group & $\mathrm{G} 1$ & $\mathrm{G} 2$ \\
\hline Male/Female & $7 / 13$ & $7 / 6$ \\
Age (years) * & $42.8 \pm 12.3$ & $39.2 \pm 10.8$ \\
BMl (kg.m ${ }^{-2}$ ) & $24.6 \pm 4.6$ & $25.2 \pm 3.3$ \\
\hline
\end{tabular}

*Values expressed in Mean \pm SD

$T_{4} / T_{1}$ ratio records obtained from initial response to 5 or 7 monitoring minutes are shown in figure 1 . There has been no fade detection in any monitoring interval, considering analyzed variables.

Intubation conditions were classified and compared according to clinical scale in $\mathrm{G} 1$ and $\mathrm{G} 2$ and there were no statistically significant differences between groups $(p=0.64)$ (Table II and Figure 2). The vast majority of patients (73.3\%) have presented clinically acceptable tracheal intubation conditions. From variables implying clinically unacceptable tracheal intubation conditions, the most important for tracheal intubation maneuvers are related to difficult laryngoscopy and closed vocal cords. These conditions were observed in 2 $\mathrm{G} 1$ and $1 \mathrm{G} 2$ patients.

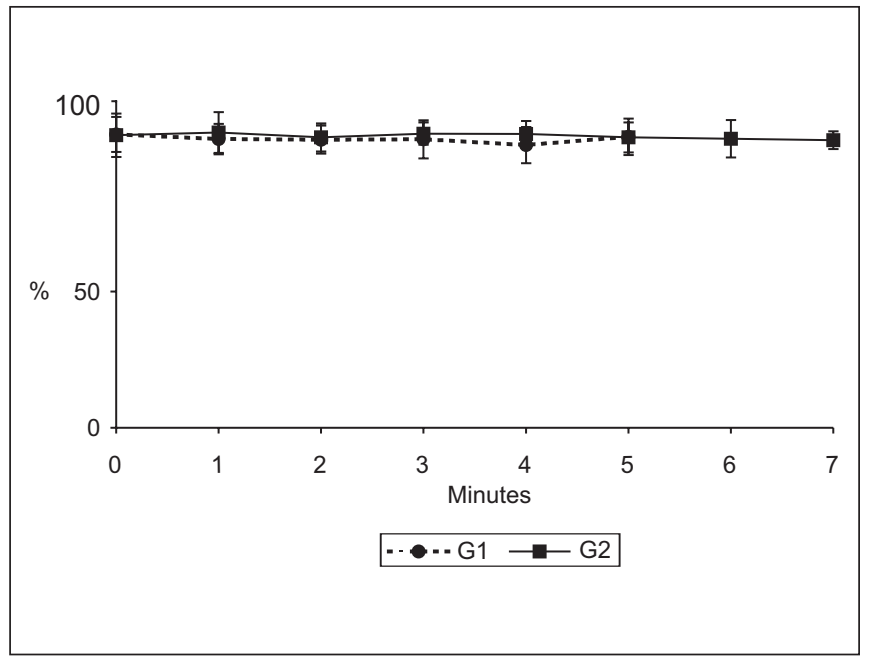

Figure 1 - Mean $T_{4} / T_{1}$ Ratio during Monitoring Interval in G1 and G2

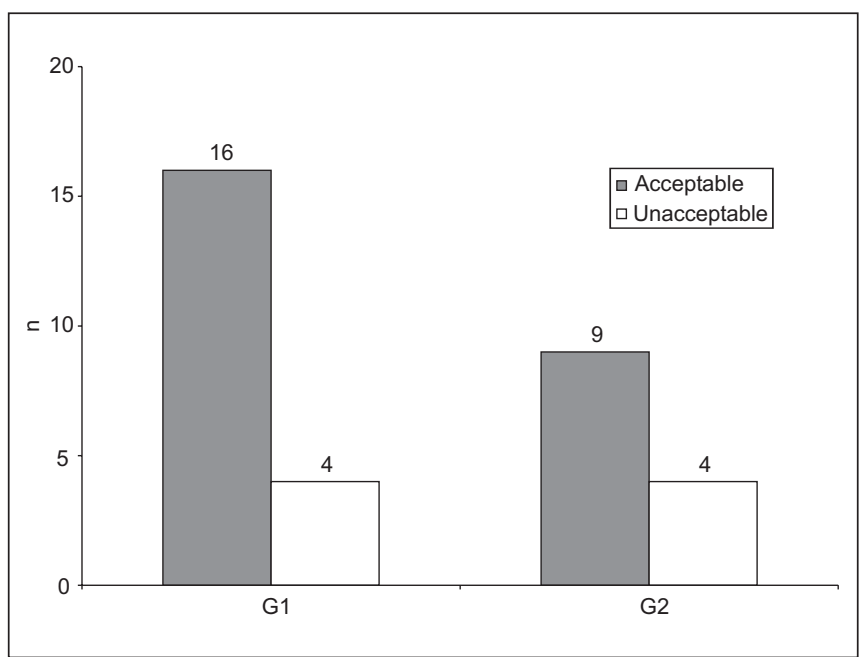

Figure 2 - Clinical Tracheal Intubation Conditions in G1 and G2

Table II - Number of Patients in G1 and G2 (G1/G2) Classified According to Clinical Conditions at Tracheal Intubation. Adapted from Viby-Mogensen et al. ${ }^{7}$

\begin{tabular}{|c|c|c|c|}
\hline \multirow[t]{2}{*}{ Variables } & \multicolumn{2}{|c|}{ Clinically Acceptable } & \multirow{2}{*}{$\frac{\text { Clinically Unacceptable }}{\text { Poor }}$} \\
\hline & Excellent & Good & \\
\hline Laryngoscopy & $\begin{array}{l}\text { Easy } \\
16 / 12\end{array}$ & $\begin{array}{l}\text { Regular } \\
2 / 2\end{array}$ & $\begin{array}{l}\text { Difficult } \\
1 / 0\end{array}$ \\
\hline \multicolumn{4}{|l|}{ Vocal Cords } \\
\hline Position & $\begin{array}{l}\text { Abducted } \\
12 / 10\end{array}$ & $\begin{array}{c}\text { Intermediate } \\
5 / 2\end{array}$ & $\begin{array}{c}\text { Closed } \\
0 / 1\end{array}$ \\
\hline Movement & $\begin{array}{l}\text { None } \\
16 / 13\end{array}$ & $\begin{array}{l}\text { Present } \\
\quad 1 / 1\end{array}$ & $\begin{array}{l}\text { Closed vocal cords } \\
2 / 1\end{array}$ \\
\hline \multicolumn{4}{|l|}{ Reaction to tube/cuff } \\
\hline With limb movements & $\begin{array}{l}\text { None } \\
11 / 8\end{array}$ & $\begin{array}{l}\text { Mild } \\
5 / 5\end{array}$ & $\begin{array}{l}\text { Strong } \\
2 / 1\end{array}$ \\
\hline With cough & $\begin{array}{l}\text { None } \\
4 / 4\end{array}$ & $\begin{array}{l}\text { With diaphragm movement } \\
12 / 8\end{array}$ & $\begin{array}{c}\text { Sustained }>10 \mathrm{sec} \\
2 / 2\end{array}$ \\
\hline
\end{tabular}




\section{DISCUSSION}

Data calling most our attention in this study was the absence of orbicularis oculi fade after priming dose.

Muscle fade may be clinically evaluated or by neuromuscular function monitoring. Among other symptoms, it is clinically manifested by blurred vision and difficult swallowing ${ }^{8}$ and there are bronchoaspiration reports after the priming dose 9,10 . When using instrumental methods, fade is considered when $T_{4} / T_{1}$ ratio is below 1 . With accelerometry, Kopman et al. ${ }^{11}$ state that $T_{4} / T_{1}$ ratio below 0.9 should be interpreted as potential incomplete neuromuscular recovery, and not below 0.7 as previously preconized.

The priming principle is based on pharmacological principle in two different sites: the first involving pre-synaptic action and the second post-junctional effect ${ }^{12}$. The pre-synaptic occupation of neuronal nicotinic receptors by neuromuscular blockers implies acetylcholine release decrease and, as a consequence, the second neuromuscular blocker dose induces faster paralysis. The authors ${ }^{13}$, however, have shown through neuromuscular transmission monitoring, that the presence of fade is not essential for the efficacy of the method. Afterward, in an experimental study ${ }^{14}$, the behavior of a nerve-muscle preparation in the presence of priming dose was evaluated and the clear conclusion was that the efficacy of the priming principle was related to drug concentration in the neuromuscular junction at the end of the priming interval and not exactly to the pre-synaptic effect.

Our objective in checking fade was not to justify principle's mechanism, but to identify an undesirable effect of the method, which would add serious complication risks, such as bronchoaspiration.

The post-junctional action indicates that firstly injected NMB molecules occupy muscle nicotinic receptors in a way that, with the second dose there is immediate relaxation because "safety margin" is overcome ${ }^{15}$. "Neuromuscular transmission safety margin" is a concept stating that to be neuromuscular function impairment it is necessary the occupation of at least $75 \%$ of neuromuscular junction receptors. This number of occupied receptors, in principle, should not be reached by the priming dose, since the objective is not muscle relaxation.

The dose needed for $75 \%$ receptors occupation is variable. Neuromuscular function monitoring, however, is unable to evaluate receptors occupation in the endplate, so that neither clinically nor with nerve stimulators it is possible to determine when safety margin is overcome, unless in its extreme manifestation with muscle relaxation.

There is much controversy about the magnitude of the priming dose to be used. Some authors ${ }^{2,8,16,17}$ mention that doses equal to or above $10 \%$ of the effective $95\left(D_{95}\right)$ dose would bring fade risk. Recently, Kopman et al. ${ }^{18}$ have published, in a study with computer emulation, that the $10 \% D_{95}$ dose would seldom cause fade, but that 1 out of 50 patients would present fade with $10 \%$ of the tracheal intubation dose. Our study has confirmed the observations about the safety of
$10 \% \mathrm{DE}_{95}$ priming dose since fade was not seen in any patient.

Another important data was that there have been no statistically significant differences between groups in tracheal intubation conditions when interval between doses was changed.

According to the literature, neuromuscular blockers onset after non-depolarizing agents is inversely proportional to the dose used ${ }^{19}$, but for sub-paralyzing doses, time to reach peak effect is relatively constant. It is currently known that this time is approximately 6 minutes for cisatracurium, 3 minutes for rocuronium and 5 minutes for vecuronium ${ }^{18}$. For atracurium, it is estimated to be $5.91 \pm 0.34$ minutes $^{13}$, time in which priming dose effect has maximum benefit in shortening neuromuscular block onset and in promoting better intubation conditions. Since tracheal intubation success rate in $\mathrm{G} 1$ and $\mathrm{G} 2$ was statistically similar, this study suggests that 5 minutes is an adequate interval to be used between atracurium doses.

Tracheal intubation conditions in this study were in general acceptable 1 minute after complementary atracurium dose. Similar values were described by Schwarz et al. ${ }^{3}$, using several priming dose modalities.

Tracheal intubation was considered clinically unacceptable in 8 cases, although we were able to intubate all patients. We have attributed this high number of patients reacting to tube/cuff to the relatively long monitoring time under superficial anesthesia. Naguib et al. ${ }^{20}$ have referred tracheal intubation conditions improvement after priming dose in a study similar to ours, using thiopental increments before intubation and confirming the concept that muscle relaxing drugs are not the single factor needed for the success of laryngoscopy and tracheal intubation maneuvers ${ }^{21}$. The absence of fade with the dose used, and not always satisfactory tracheal intubation conditions point exactly to the two questionable priming principle points: the dose protecting airway against bronchoaspiration may not be sufficient for tracheal intubation success. Although being able to intubate all cases, the presence of events such as closed vocal cords and difficult laryngoscopy should be appreciated and considered failures and limitations to the method. In patients at risk for bronchoaspiration, the risks this technique may impose should be thoroughly evaluated.

In conclusion, one may state that priming $0.02 \mathrm{mg} \cdot \mathrm{kg}^{-1}$ atracurium dose $\left(10 \% \mathrm{DE}_{95}\right)$ does not induce orbicularis oculi fade in up to 7 minutes and that there is no statistical difference in tracheal intubation conditions when 5 or 7 minutes are waited between priming and intubation doses.

\section{REFERÊNCIAS - REFERENCES}

01. Chingmuh I - Succinylcholine update. Curr Opin Anaesthesiol, 1993;6:709-714.

02. Aziz L, Jahangir SM, Choudhury SN et al - The effect of priming with vecuronium and rocuronium on young and elderly patients. Anesth Analg, 1997;85:663-666. 
03. Schwarz S, Ilias W, Lackner F et al - Rapid tracheal intubation with vecuronium: the priming principle. Anesthesiology, 1985;62:388-391.

04. Miller RD - The priming principle. Anesthesiology, 1985;62: 381-382.

05. Donati F, Meistelman C, Plaud B - Vecuronium neuromuscular blockade at the diaphragm, the orbicularis oculi, and adductor pollicis muscles. Anesthesiology, 1990;73:870-875.

06. Debaene B, Beaussier M, Meistelman C et al - Monitoring the onset of neuromuscular block at the orbicularis oculi can predict good intubating conditions during atracurium-induced neuromuscular block. Anesth Analg, 1995;80:360-363.

07. Viby-Mogensen J, Engbaek J, Eriksson Ll et al - Good clinical research practice (GCRP) in pharmacodynamic studies of neuromuscular blocking agents. Acta Anaesthesiol Scand, 1996;40:59-74

08. Mahajan RP, Laverty J - Lung function after vecuronium pretreatment in young, healthy patients. $\mathrm{Br} \mathrm{J}$ Anaesth, 1992;69: 318-319.

09. Musich J, Walts LF - Pulmonary aspiration after a priming dose of vecuronium. Anesthesiology, 1986;64:517-519.

10. Engbaek J, Howardy-Hansen $\mathrm{P}$, Ording $\mathrm{H}$ - Precurarization with vecuronium and pancuronium in awake, healthy volunteers: the influence on neuromuscular transmission and pulmonary function. Acta Anaesthesiol Scand, 1985;29:117-120.

11. Kopman AF, Klewicka MM, Neuman GG - The relationship between acceleromyographic train-of-four fade and single twitch depression. Anesthesiology, 2002;96:583-587.

12. Jones RM - The priming principle: how does it work and should we be using it? $\mathrm{Br} \mathrm{J}$ Anaesth, 1989;63:1-3

13. Naguib M, Abdulatif M, Gyasi HK et al - The pattern of train-of-four fade after atracurium: influence of different priming doses. Anesth Analg, 1987;66:427-430.

14. Storella RJ, Jaffe J, Mehr E et al - In vitro investigation of the priming principle for rapid neuromuscular block. Br J Anaesth, 1989;62:478-482.

15. Paton WD, Waud DR - The margin of safety of neuromuscular transmission. J Physiol, 1967;191:59-90.

16. Donati $F$ - The priming saga: where do we stand now? Can J Anaesth, 1988;35:1-4

17. Glass PS, Wilson W, Mace JA et al - Is the priming principle both effective and safe? Anesth Analg, 1989;68:127-134.

18. Kopman AF, Khan NA, Neuman GG - Precurarization and priming: a theoretical analysis of safety and timing. Anesth Analg, 2001;93:1253-1236.

19. Healy TE, Pugh ND, Kay B et al - Atracurium and vecuronium: effect of dose on the time of onset. $\mathrm{Br} J$ Anaesth, 1986;58: 620-624.

20. Naguib M, Abdulatif M, Gyasi HK et al - Priming with atracurium: improving intubating conditions with additional doses of thiopental. Anesth Analg, 1986;65:1295-1299.

21. Griffith KE, Joshi GP, Whitman PF et al - Priming with rocuronium accelerates the onset of neuromuscular blockade. J Clin Anesth, 1997;9:204-207.

\section{RESUMEN}

Locks GF, Almeida MCS - Dosis Preparatoria de Atracurio: Comparación de la Fatiga en el Músculo Orbicular del Ojo y Condiciones de Intubación Orotraqueal

JUSTIFICATIVA Y OBJETIVOS: El método de la dosis preparatoria (priming principle) consiste en la inyección de una pequeña dosis del bloqueador neuromuscular minutos antes de la administración de lo que queda de la dosis preconizada para intubación traqueal para encortar el inicio de acción de los bloqueadores neuromusculares (BNM) adespolarizantes. Existe, sin embargo, posibilidad de fatiga muscular $y$ broncoaspiración. De especial interés para las maniobras de intubación orotraqueal y protección de las vías aéreas, son los músculos de la laringe. Como su monitorización directa impone dificultades técnicas, se demostró que el músculo orbicular del ojo presenta correlación con los músculos laríngeos cuanto a la sensibilidad a los BNM. El objetivo de esta pesquisa es evaluar si hay fatiga del músculo orbicular ocular después del uso de la dosis preparatoria de atracúrio y comparar las condiciones clínicas de intubación traqueal después de dos intervalos entre las dosis.

MÉTODO: Fueron seleccionados 35 pacientes adultos, estado físico ASA I o II, sin factores de riesgo para broncoaspiración, sometidos a cirugía electiva. Fue inducida anestesia general con alfentanil y propofol y el paciente fue ventilado manualmente bajo máscara. En seguida, fueron posicionados los electrodos para estimular el ramo temporal del nervio facial y el transductor de aceleración en el músculo orbicular del ojo. Se administró dosis preparatoria de atracúrio $\left(0,02 \mathrm{mg}^{\mathrm{kg}} \mathrm{kg}^{-1}\right)$ y la relación $T_{4} / T_{1}$ fue evaluada a cada minuto durante 5 minutos en 20 casos (G1) y 7 minutos en 13 casos (G2). Después de este intervalo, se administró la dosis complementar de atracúrio $\left(0,5 \mathrm{mg} \cdot \mathrm{kg}^{-1}\right)$ y se procedió a la intubación orotraqueal después de un minuto. Fue considerada fatiga clínicamente importante la relación $T_{4} / T_{1}$ inferior a 0,9.

RESULTADOS: Ningún paciente presentó fatiga durante el intervalo de monitorización. En $80 \%$ y $69 \%$ de los pacientes de G1 ó G2, respectivamente, las condiciones de intubación traqueal fueron consideradas clínicamente aceptables ( $p>$ $0,05)$.

CONCLUSIONES: La dosis preparatoria de atracúrio $(0,02$ mg. $\mathrm{kg}^{-1}$ ) no causa fatiga en el músculo orbicular del ojo y no hay diferencia entre esperar 5 ó 7 minutos como intervalo entre las dosis del bloqueador neuromuscular. 